\title{
General Equations for the Induced Phase Shift in Resonant Electrooptic Modulators
}

\author{
C. Collado, O. Menéndez, M. C. Santos, J. Mateu, and J. M. O’Callaghan
}

\begin{abstract}
We derive general equations to calculate the electrooptically induced optical phase shift in resonantly enhanced modulators. The equations are useful in designing modulator electrodes with optimal length and optimal mismatch between electrical and optical velocities. We demonstrate the existence of an optimal velocity mismatch depending on the resonant mode used.
\end{abstract}

Index Terms-Electrooptical modulator, resonant modulator, resonant electrode, optimal walkoff.

\section{INTRODUCTION}

A BROAD scope of analog applications for electrooptical modulators has emerged with the advent of radio-overfiber systems [1]. The modulators used in these narrow-band applications may benefit from the electric field enhancement that results from using resonant electrodes [2]. This enhancement reduces the power of the modulating source required to drive the device at the expense of a reduced operation bandwidth around the resonance frequency. A variety of planar resonant modulators has been proposed and experimentally demonstrated, showing significant improvements in modulation efficiency [2]-[4]. These devices were designed using either numerical methods or analytical equations specific for a given modulator configuration. However, a general study relating modulation depth with the parameters of a resonant electrode and the power of the modulating source is still missing. Without that study, important issues like the optimal ratio of optical and electrical velocities are difficult to resolve, especially when it comes to approaching a novel modulator design.

In this letter, we derive a general equation for the optical phase shift induced by the electrical signal in a resonant modulator. This equation is applicable to all types of resonant modulators and provides a basis for comparing among various topologies, predicting the performance of novel designs, establishing the relationships among all the parameters involved in the modulation process, and identifying the optimum design rules.

Manuscript received March 30, 2004; revised August 31, 2004. This work was supported by the Spanish Ministry of Science and Technology through Project TEC2004-06194-C02 and by Generalitat de Catalunya (DURSI) through Grant 2001 SGR 00266.

C. Collado, O. Menéndez, and M. C. Santos are with Universitat Politècnica de Catalunya (UPC)-TSC, Barcelona 08034,Spain (e-mail: collado@tsc.upc.es; omenendez@tsc.upc.es; santos@tsc.upc.es).

J. Mateu is with NEXUS I-CTTC-Centre Tecnològic de Telecomunicacions de Catalunya, Barcelona 08034, Spain (e-mail: jordi.mateu@cttc.es).

J. M. O'Callaghan is with Universitat Politècnica de Catalunya (UPC)-TSC, Barcelona 08034, Spain (e-mail: joano@tsc.upc.es).

Digital Object Identifier 10.1109/LPT.2004.839465
The letter also shows the validation of the general equation by applying it to a particular modulator [4] that can be analyzed using a specific numerical technique.

\section{EFFICIENCY OF MODULATION IN RESONANT MODULATORS}

\section{A. Formulation}

In a transmission line electrooptical modulator, a guided optical beam runs parallel to electrical electrodes supporting a voltage $v(z, t)$. For photons incident at time $t=t_{0}$ at the position $z=-L / 2$, the induced phase shift $\Delta \Phi\left(t_{0}\right)$ over an interaction length $L$ is given by [2]

$$
\Delta \Phi\left(t_{0}\right)=-\frac{\pi N_{0}^{3} r \Gamma}{\lambda_{0} G} \int_{-L / 2}^{L / 2} v\left(z, t_{0}+N_{0} z / c\right) d z
$$

where $\lambda_{0}$ is the free-space optical wavelength, $c$ the vacuum light velocity, $N_{0}$ and $r$ are the optical refractive index and electrooptic coefficient, and $\Gamma$ and $G$ are the optical-electrical mode field overlap integral factor and interelectrode gap respectively.

In a resonant configuration of the electrodes, when the resonator is fed at resonance with a single-tone source of frequency $\omega_{r}$, the voltage $v(z, t)$ can be written as

$$
v(z, t)=V_{m} \cos \left(N_{e} \omega_{r} z / c+\Phi_{z}\right) \cos \left(\omega_{r} t\right)
$$

where $N_{e}$ denotes the effective index for the propagation mode supported by the electrodes [5], $V_{m}$ is the peak voltage, and $\Phi_{z}$ determines the position of the voltage maximum, which depends on the end terminations of the transmission line electrodes and the order of the resonant mode. The standing-wave pattern in (2) assumes that losses in the electrodes do not significantly affect the voltage distribution along the line.

Microwave theory has developed solutions to relate the amplitude of the fields in a resonator with the power of the source driving it. These solutions can be applied in our case to relate the voltage $V_{m}$ in (2) to the source voltage $V_{s}$. The key assumption is that the source is coupled to the resonator through a lossless network. This network may provide impedance matching to the source, so that all its available power is transferred to the resonator. In this condition, the peak voltage $V_{m}$ can be related to $V_{s}$ (see the Appendix)

$$
V_{m}=\left(V_{s} / 2\right) G_{v} \sqrt{N_{e} / n}
$$

where $n=L / \lambda_{e}$ quantifies the resonant mode order $(n=$ $1 / 4, n=1 / 2, n=1 \ldots)$ being $\lambda_{e}=(2 \pi c) /\left(\omega_{r} N_{e}\right)$, the guided electrical wavelength [5]. The dimensionless parameter 
$G_{v}$ depends exclusively on electrical magnitudes (see the Appendix) and is defined as $G_{v}=\sqrt{Z_{0} /\left(\alpha Z_{s} \lambda_{e, 0}\right)}$, where $\lambda_{e, 0}$ is the vacuum wavelength, $Z_{0}$ is the characteristic impedance of the transmission line, and $\alpha$ is its attenuation constant given in $\mathrm{Np} / \mathrm{m}$. Even though (3) assumes critical coupling-impedance matching at resonance- - the equation can be easily extended to find $V_{m}$ for other coupling conditions (see the Appendix). The Appendix also shows how (3) can be extended for frequencies that are close to resonance, but not necessarily equal to the resonant frequency.

The difficulty in setting the basic device parameters without combining (1)-(3) in a single equation is exemplified in the election of the electrode length $L$ for a given frequency of operation $\left(\omega_{r}\right)$. According to (1), a long interaction length $L$ might lead to high phase shift $\Delta \Phi\left(t_{0}\right)$. However, a long electrode requires a low $N_{e}$ or making the electrode several quarter wavelengths long (high $n$ ). Both options reduce $V_{m}$ according to (3) and tend to decrease $\Delta \Phi\left(t_{0}\right)$.

In order to find the optimal configuration, it is necessary to solve (1) for the different possible standing-wave patterns. We can do this in a unified way by substituting (2) and (3) into (1)

$$
\begin{aligned}
\Delta \Phi\left(t_{0}\right)= & A\left(V_{s} / 2\right) G_{v}\left(n \Psi_{n}\right)^{-1 / 2} \\
& \cdot \int_{-L / 2}^{L / 2} \cos \left(z N_{0} \omega_{r} /\left(c \Psi_{n}\right)+\Phi_{z}\right) \\
& \times \cos \left(\omega_{r} t_{0}+N_{0} \omega_{r} z / c\right) d z
\end{aligned}
$$

where $A$ is defined as $A=-\pi N_{0}^{7 / 2} r \Gamma / \lambda_{0} G$ and the velocity mismatch factor $\Psi_{n}=N_{0} / N_{e}$ quantifies the walkoff between electrical and optical waves. Evaluating the integral in (4) along the limits $\pm L / 2= \pm n \pi c / N_{e} \omega_{r}$, one can calculate the maximum phase change $|\Delta \Phi|$, as shown in (5) at the bottom of the page.

This formula is the main contribution of this work since it directly connects $|\Delta \Phi|$ with the velocity mismatch factor $\Psi_{n}$, for several resonating modes characterized through the values of $\Phi_{z}$ and $n$.

Fig. 1 shows the magnitude of the phase shift $\left(\left|\Delta \Phi_{N}\right|\right)$ normalized to $A V_{s} G_{v} \lambda_{e, 0} / 2 \pi N_{0}$, as a function of the velocity mismatch factor $\Psi_{n}$ for three resonators. This figure, or a similar one including other topologies, is useful either for choosing the most adequate resonant topology for a given velocity mismatch, or for finding the optimum velocity mismatch to maximize the modulation efficiency given an electrical standing-wave pattern.

Table I summarizes other relevant results. It shows, for a set of representative resonator configurations, the values of $\left|\Delta \Phi_{N}\right|$ obtained with a typical walkoff $\Psi_{n}=0.8$ in $\mathrm{LiNbO}_{3}$ modulators [4], together with the optimum mismatch $\Psi_{o}$ that maximizes the induced phase shift and the corresponding maximum.

From these data, we conclude that $\left|\Delta \Phi_{N}\right|$ tends to increase for higher resonant modes since the increase of the interaction

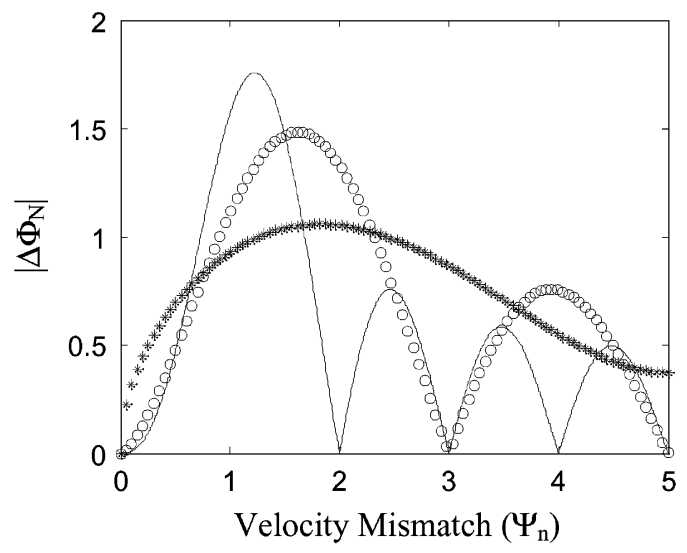

Fig. 1. Normalized $\left|\Delta \Phi_{N}\right|$ as a function of the velocity mismatch for three resonators, $L=\lambda_{e} / 4$ short-open ended (asterisks), $L=\lambda_{e} / 2$ open ended (circles), and $L=\lambda_{e}$ open ended (continuous line).

TABLE I

MAXIMUM PHASE SHIFT IN DIFFERENT TyPES OF MODULATORS

\begin{tabular}{cccccc}
\hline \hline Length & Ended & $\Phi_{z}$ & $\Delta \Phi_{\mathrm{N}} \mid$ if $\Psi_{\mathrm{n}}=0.8$ & $\Psi_{\mathrm{o}}$ & $\Delta \Phi_{\mathrm{N}} \mid$ if $\Psi_{\mathrm{o}}$ \\
\hline$\lambda_{\mathrm{e}} / 4$ & React. & 0 & 1.19 & 1.6 & 1.39 \\
$\lambda_{\mathrm{e}} / 4$ & Short- & $\pm \pi / 4$ & 0.85 & 1.8 & 1.06 \\
& open & & & & \\
$\lambda_{\mathrm{e}} / 2$ & Short & 0 & 1.09 & 1 & 1.11 \\
$\lambda_{\mathrm{e}} / 2$ & Open & $-\pi / 2$ & 0.87 & 1.6 & 1.48 \\
$\lambda_{\mathrm{e}}$ & Short & $-\pi / 2$ & 1.46 & 1 & 1.57 \\
$\lambda_{\mathrm{e}}$ & Open & 0 & 1.17 & 1.2 & 1.76 \\
\hline \hline
\end{tabular}

length compensates the decrease of the maximum voltage in the line. Furthermore, open-ended topologies for $\lambda_{e} / 2$ and $\lambda_{e}$ resonators result more efficient than short-ended ones at the optimum walkoff but not for a typical walkoff in $\mathrm{LiNbO}_{3}$ modulators. That is, the most efficient topology depends on the feasible walkoff for a given technology.

\section{B. Formulation Check}

We have checked (5) with the numerical technique described in [4] which is based on transmission line theory and is validated through experimental measurements. For all modulators tested (those of Table I), there is accurate coincidence when losses of the transmission line are low. Otherwise, if the losses are very high, the accuracy of (5) decreases since the voltage distribution deviates from the assumed standing wave pattern in (2). To illustrate this effect and give an estimate of the error, we particularize (5) for a open-ended $\lambda_{e} / 2$ resonator $\left(\Phi_{Z}=-\pi / 2\right.$ and $n=1 / 2)$

$$
|\Delta \Phi(\omega)|=\left|A V_{s} G_{v}(\omega) \sqrt{2} \frac{\lambda_{e, 0}}{2 \pi N_{0}} \frac{\Psi_{n}^{3 / 2}}{\Psi_{n}^{2}-1} \cos \left(\frac{\pi \Psi_{n}}{2}\right)\right| .
$$

In this equation, the parameter $G_{v}$ of (5) has been replaced by $G_{v}(\omega)=G_{v} \sqrt{F(\omega)}$, being $F(\omega)$ a term which accounts for

$$
\begin{array}{r}
|\Delta \Phi|=\left|A V_{s} G_{v} \frac{\lambda_{e, 0}}{2 \pi N_{0}} \sqrt{\frac{1}{n}} \frac{\sqrt{\Psi_{n}}}{\left(\Psi_{n}^{2}-1\right)} \sin \left(n \pi \Psi_{n}\right) \cos (n \pi) \cos \left(\Phi_{z}\right)\right| \\
\cdot \sqrt{\left[\Psi_{n}-\cot \left(n \pi \Psi_{n}\right) \tan (n \pi)\right]^{2}+\left[1-\Psi_{n} \cot \left(n \pi \Psi_{n}\right) \tan (n \pi)\right]^{2} \tan ^{2}\left(\Phi_{z}\right)}
\end{array}
$$




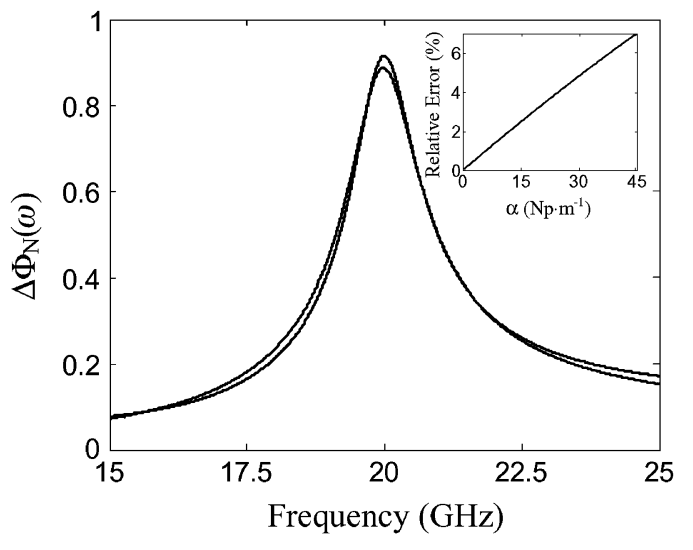

Fig. 2. Comparison between $\left|\Delta \Phi_{N}(\omega)\right|$ from (6) (dashed-dotted line) with that obtained using [4] (continuous line). The inset shows the relative error between both methods as a function of $\alpha$.

the frequency response of the resonator (see the Appendix). The addition of this term extends (5) — we denote $|\Delta \Phi(\omega)|$ instead of $|\Delta \Phi|$ - and gives the response of the induced phase shift around the resonance frequency.

Fig. 2 compares the normalized $\Delta \Phi_{N}(\omega)$ of (6) with that obtained using the simulation method described in [4]. This example assumes a $20-\mathrm{GHz}$ resonator with realistic parameters taken from [4]: $Z_{s}=50 \Omega, Z_{0}=35 \Omega, \Psi_{n}=0.8$, and $\alpha=18 \mathrm{~Np} \cdot \mathrm{m}^{-1}$.

The agreement between both traces is quite good and the estimated maximum value using the numerical technique of [4] is only $3 \%$ greater than that from (6). The inset shows the relative error between both methods as a function of $\alpha$, showing that the proposed formulation is a very good approximation for the usual values of losses in a conventional transmission line.

\section{CONCLUSION}

We have derived equations for the analysis of resonantly enhanced electrooptical modulators. These equations are useful in setting the length of the resonant electrode and the ratio between electrical and optical velocities. We show that it is not always necessary to match these velocities to maximize the efficiency of the modulator. This conclusion is especially important for GaAs modulators where the velocity of the electrical signal is usually greater than that of the optical signal. For example, [6] gives $N_{o}=3.2$ and $N_{e}=2.6$ for a conventional coplanar strip, which implies a walkoff parameter $\Psi_{n}=1.23$ closer to the optimal walkoff of most topologies of Table I than the velocity matching condition $\Psi_{n}=1$. Therefore, designing the electrodes to match velocities could be unnecessary, even counterproductive.

Finally, the equations derived in this letter are not restricted to transmission line modulators, since (2) is applicable to the electric field of any resonator that results from the longitudinal propagation of a guided wave.

\section{APPENDIX}

Using microwave analysis [5], the maximum voltage at the resonant electrode $V_{m}$ can be related to the voltage source $V_{s}$

$$
V_{m}=\left(V_{s} / 2\right) G_{v} \sqrt{N_{e} / n} \sqrt{4 k /(k+1)^{2}} \sqrt{F(\omega)}
$$

where $F(\omega)=\left(1+\Delta^{2}(\omega) Q_{L}^{2}\right)^{-1}$ accounts for the frequency dependence close to resonance $\left(\Delta(\omega)=1-\omega_{r}^{2} / \omega^{2}\right)$, being $Q_{L}$ the loaded quality factor, $\kappa$ the coupling coefficient that relates the dissipated power inside the resonator with the available power of the source [5], and $G_{V}$ a constant given by $G_{v}=$ $\sqrt{Z_{o} /\left(\alpha Z_{s} \lambda_{e, 0}\right)}$.

Assuming critical coupling at resonance, that is $\kappa=1$ and $\Delta(\omega)=0$ [5], the previous expression can be reduced to

$$
V_{m}=\left(V_{s} / 2\right) G_{v} \sqrt{N_{e} / n}
$$

\section{REFERENCES}

[1] A. Vilcot, B. Cabon, and J. Chazelas, Microwave Photonics: From Components to Applications and Systems. Norwell, MA: Kluwer, 2003.

[2] G. K. Gopalakrishnan and W. K. Burns, "Performance and modeling of resonantly enhanced $\mathrm{LiNbO}_{3}$ modulators for low-loss analog fiberoptic links," IEEE Trans. Microwave Theory Tech., vol. 42, no. 12, pp. 2650-2656, Dec. 1994.

[3] K. Yoshida, Y. Kanda, H. Yoshihara, H. Kanaya, S. Shinkai, and M. Ishitobi, "Design and performance of superconducting circuits for $\mathrm{LiNbO}_{3}$ optical modulator and switch," IEEE Trans. Appl. Supercond., vol. 13, no. 2, pp. 1127-1130, Jun. 2003.

[4] R. Krähenbühl and M. M. Howerton, "Investigations on short-pathlength high-speed optical modulators in $\mathrm{LiNbO}_{3}$ with resonant-type electrodes," J. Lightw. Technol., vol. 19, no. 9, pp. 1287-1297, Sep. 2001.

[5] D. M. Pozar, Microwave Engineering. New York: Wiley, 1998.

[6] N. A. Jaeger, F. Rahmatian, H. Kato, R. James, E. Berolo, and Z. K. Lee, "Velocity-matched electrodes for compound semiconductor travelling-wave electrooptic modulators: Experimental results," IEEE Microw. Guided Wave Lett., vol. 6, no. 2, pp. 82-84, Feb. 1996. 